\title{
Information et démocratie municipale : les glissements de l'expérience québécoise
}

Alain Lavigne

\section{OpenEdition}

1 Journals

Édition électronique

URL : http://journals.openedition.org/communicationorganisation/1742

DOI : 10.4000/communicationorganisation. 1742

ISSN : $1775-3546$

Éditeur

Presses universitaires de Bordeaux

Édition imprimée

Date de publication : 1 novembre 1994

ISSN : 1168-5549

Référence électronique

Alain Lavigne, «Information et démocratie municipale : les glissements de l'expérience québécoise », Communication et organisation [En ligne], 6 | 1994, mis en ligne le 26 mars 2012, consulté le 20 avril 2019. URL : http://journals.openedition.org/communicationorganisation/1742 ; DOI : 10.4000/ communicationorganisation. 1742

Ce document a été généré automatiquement le 20 avril 2019

(c) Presses universitaires de Bordeaux 


\title{
Information et démocratie municipale : les glissements de l'expérience québécoise
}

\author{
Alain Lavigne
}

1 Depuis quelques décennies, l'enjeu démocratique constitue l'une des valeurs phares du palier municipal dans plusieurs pays occidentaux. De fait, à titre de niveau de gouvernement le plus près de citoyens, le palier municipal a suscité ici et là diverses réformes visant à favoriser l'idéal démocratique, notamment en matière d'accès à l'information et de consultation des citoyens.

2 Dans le présent article, notre contribution vise à discuter de cette problématique particulière à travers l'expérience québécoise des quinze dernières années. La Loi sur la démocratie municipale de 1980 et la Loi sur l'accès aux documents des organismes publics et sur la protection des renseignements personnels de 1982 ont en effet imposé aux quelque 1500 municipalités du Québec de nouvelles règles du jeu en matière d'information.

3 L'hypothèse que nous voulons évaluer ici est que les nouvelles mesures d'accès à l'information et de consultation des citoyens n'ont pas changé de façon substantielle les ressources de pouvoir des principaux acteurs du système municipal québécois, à savoir les élus, les fonctionnaires, les intermédiaires (journalistes et groupes d'intérêt) et les « simples » citoyens.

4 Pour ce faire, nous présentons un aperçu des écrits portant sur l'information municipale comme ressource de pouvoir. Par la suite, nous élaborons sur le contexte d'intervention et sur certains glissements de l'expérience québécoise en matière d'accès à l'information et de consultation des citoyens. Enfin, nous en évaluons les principales conséquences sur le système municipal actuel. 


\section{Information municipale : une ressource de pouvoir}

5 Au cours des dernières décennies, les écrits français et québécois, plus particulièrement, ont associé l'information municipale à une ressource de pouvoir. Ces travaux empruntent plus ou moins explicitement quatre angles d'analyse distincts : politique, administratif, celui de la presse, enfin, celui des groupes et des citoyens.

6 Les auteurs qui analysent, dans un contexte français, l'information municipale comme une ressource politique insistent sur le rôle et le discours (Chanel, 1980) des élus dans le contrôle de cette ressource. L'information est ainsi vue comme un enjeu important parce qu'elle constitue un instrument de pouvoir pour les élus (Montergnole, $1980: 87$ ). Selon Lagroye (1980), l'information municipale tend à maintenir le consensus et à légitimer les actions entreprises localement par l'administration et les élus. L'objectif premier est souvent de convaincre les électeurs qu'il y a bien une politique municipale et que les réalisations ne sont pas le fruit du hasard. Le danger est alors celui d'une communication se contentant de proclamer la légitimité des élus à travers leurs réalisations, sans engager un dialogue avec les électeurs sur les questions de fond (Rangeon, 1991 :110).

7 Pour sa part, Pailliart (1982, 1984, 1989, 1990, 1993) soutient que cette information, proclamée au service de la démocratie demeure la chasse gardée d'un seul personnage : le maire. D'après l'auteur, dans la gestion de leur

8 ville, qui se rapproche de plus en plus du modèle commercial, les maires utilisent tous les moyens modernes de communication. Ainsi, malgré une certaine professionnalisation, la dépendance vis-à-vis du pouvoir politique reste manifeste.

«Le service information-communication a, en grande partie, comme interlocuteur principal et comme responsable, le directeur de cabinet du maire ou le maire luimême. Plus rarement, il dépend de l'adjoint à la communication. De toute façon, en cas de litige ou de discussion, c'est au cabinet du maire ou au maire lui-même que revient le pouvoir d'arbitrage. » (Pailliart, 1993 :98)

Dans cette perspective, Miège (1989) affirme que la communication est devenue une affaire de "professionnels». Plus que jamais, affirme l'auteur, la communication participe à la formation et au renforcement du positionnement des maires. De son côté, Simon (1991) rappelle que la forte présence du niveau politique dans tous les supports d'information des villes est là pour souligner qu'il s'agit toujours d'un secteur stratégique. En effet, la communication institutionnelle des collectivités est contrôlée par le détenteur du pouvoir qui peut ainsi en user pour faire connaître et valoir son action (Gerstlé, 1992 :101-102). Dans un tel contexte d'action, à la manière d'un chef d'entreprise, le «maire stratège» exerce un pouvoir fort et discrétionnaire (Padioleau, $1990: 59$ ). En référant à l'article de Lagroye, Dion (1986:186) observe, pour sa part, que la politique d'information des cinq municipalités de gauche de la banlieue parisienne qu'il a étudiées semble, avant tout, avoir été conçue pour être créatrice de consensus et susceptible d'accréditer une image du maire et des élus empreinte d'efficacité et de réussite dans l'action.

10 Au Québec, l'analyse de Andrew et al. (1975) sur l'information du programme de logement public à Hull met aussi en lumière le rôle du pouvoir politique. Les auteurs insistent pour ne pas isoler cette politique d'information particulière du processus politique plus large. En contexte électoral, Quesnel et Belley $(1991)$ ainsi que Demers $(1988,1990)$ cernent bien, pour leur part, les stratégies d'action des partis politiques pour que leurs messages soient 
relayés le plus fidèlement possible par les médias. À travers la masse des informations livrées par les médias, les électeurs peuvent se faire une idée des candidats et des options politiques en présence (Quesnel et Belley, $1991: 157)$. Dans le cas du débat télévisé L'Allier/Bertrand, Gauthier (1990) identifie d'ailleurs l'argumentation stratégique des politiciens en pleine joute électorale médiatisée. La fonction de l'argumentation stratégique est ainsi de créer des effets émotifs et de donner de la crédibilité à l'argumentation pour gagner l'adhésion de l'auditoire (Gauthier, 1990 :139).

11 Par ailleurs, quelques travaux traitent l'information municipale sous l'angle d'une ressource de pouvoir de l'acteur administratif. Selon Dion (1984:4), les fonctionnaires municipaux travaillent en principe dans des conditions précises, répartis en différentes unités ou services selon le mode bureaucratique de la hiérarchisation des postes et des fonctions. Les jeux de pouvoirs entre élus et fonctionnaires sont complexes et ambivalents, surtout en ce qui a trait à la gestion de l'information. Dion (1984:5) donne l'exemple d'un fonctionnaire qui ferait en sorte que des élus ou membres des cabinets politiques soient amenés à court-circuiter les procédures normales de l'administration, ou encore, lorsqu'un fonctionnaire organise des « fuites » d'informations au bénéfice des partis d'opposition ou directement par les médias, non pas pour venir en aide à des alliés politiques éventuels, mais afin d'améliorer sa propre position dans l'administration. Dans une perspective organisationnelle, Becquart-Leclercq (1988:23) caractérise cette dynamique locale par le concept de dualité culturelle: l'une liée à la logique élective, l'autre liée à la logique administrative. Dans ce contexte, relèvent Gravel et Petrelli (1989a, 1989b), le directeur général a un pouvoir implicite significatif en matière d'information municipale, en raison de sa position d'intermédiaire entre l'administratif et le politique.

12 Par la mise sur pied de codes déontologiques professionnels visant à ne pas confondre l'information administrative et politique, l'acteur administratif affirme également son pouvoir d'« expert » en matière d'information administrative. Au Québec, c'est dans cette perspective qu'il faut interpréter les travaux de l'Association des communicateurs municipaux, Le guide de la communication municipale (Collard et al., 1982) et Les règles de base de la communication municipale (Gagnon Hosson, 1987).

13 L'information municipale a aussi été étudiée sous l'angle du pouvoir de la presse. Celui-ci lui est consenti par son rôle de relayeur sélectif de l'information. L'analyse de Biais et Crête (1982), à propos de la presse et de la politique dans deux villes du Québec, relève cette dimension de l'information municipale. Selon les auteurs, l'influence de la presse sur les résultats des élections municipales est indirecte. Dans le processus d'information sur les affaires municipales, il est clair que l'initiative appartient aux élus et aux fonctionnaires, la presse n'étant souvent qu'une courroie de transmission (1982:65-66). Appliquant la démarche d'analyse de Black et Snow (1982) à une région du Québec, la thèse de Quenneville (1984), sur la presse écrite locale et l'ordre du jour des affaires publiques municipales en Mauricie, évalue que le pouvoir de la presse s'avère plutôt restreint. À l'égard de l'établissement de l'ordre du jour, les médias étudiés sont plutôt apparus comme des intermédiaires passifs entre leurs lecteurs et les acteurs sociaux dominants : les décideurs (1984:134).

14 Dans cet ordre d'idées, un sondage mené conjointement en 1988 par l'Union des municipalités du Québec (UMQ) et la Fédération professionnelle des journalistes du Québec (FPJQ) a fait état des relations entre les municipalités et la presse (Plourde, 1988a ; $1988 \mathrm{~b} ; 1988 \mathrm{c} ; 1988 \mathrm{~d})$. Ce sondage tend aussi à démontrer que même avec une structure 
des communications très développée, le maire joue un grand rôle, sinon l'unique rôle, dans le domaine des relations avec les médias (Plourde, 1988b :10).

Finalement, plusieurs autres auteurs ont abordé l'information municipale sous l'angle d'une ressource pour les groupes et les citoyens. L'information y est alors perçue comme une nouvelle ressource de pouvoir permettant l'émergence d'une meilleure démocratie locale. Les travaux de Beaunez $(1975 ; 1976)$, Mallet (1978), Garnier et Goldchmidt (1978), Gontcharoff $(1980 ; 1985 ; 1989 ; 1990 a ; 1990 b ; 1991)$, Josephe et Murret (1983), Castagnet (1991) Comet (1991) Revelin (1983) et Grémion (1991) peuvent être regroupés dans ce même courant d'analyse, même si ces auteurs développent la question selon des grilles plus ou moins critiques. Par exemple, certains auteurs (Garnier et Goldchmidt, 1978 :226) mettent l'accent sur les dangers que représentent les procédures de consultation et de participation mises en œuvre à l'occasion de vastes opérations d'urbanisme.

«Ces auteurs parlent d'une information au service d'une profonde 'mystification'qui consiste à persuader les citadins qu'ils peuvent agir sur 'leur cadre de vie', voire 'changer la vie'par ce biais, alors que l'un et l'autre sont modelés par des processus dont la dynamique leur échappe totalement. » (cité par Pailliart, 1993 :49)

En résumé, malgré des angles d'analyse bien distincts, retenons que les écrits français et québécois présentant l'information municipale comme ressource de pouvoir tendent à confirmer, pour la plupart, le rôle déterminant des décideurs politiques et des fonctionnaires de leur administration. Au Québec, le projet de réforme sur la démocratie municipale souhaitait précisément changer cet état de choses.

\section{La démocratisation du palier municipal}

\section{Le contexte d'intervention}

Entre les années 60 et 80, il est opportun de rappeler que la scène municipale québécoise est le théâtre de nombreux changements aux plans social et politique. Selon les historiens (Linteau et al., 1986 :500), dans l'euphorie des années 60, dans un environnement dominé par les promoteurs privés, les projets sont perçus comme des symboles de progrès. Les promoteurs arrivent alors sans trop de mal à faire accepter leurs projets par les municipalités qui se préoccupent timidement d'urbanisme, mais se limitent le plus souvent à l'adoption de règlements de zonage et à l'embellissement des espaces publics.

Au tournant des années 70 , des groupes de citoyens réagissent contre les démolitions massives et amènent graduellement les gouvernements à se préoccuper de la qualité de la vie et à faire des efforts en vue de préserver les espaces historiques. Un tel contexte social est propice à l'élaboration d'une nouvelle conception du rôle des municipalités par le gouvernement du Québec. Par de multiples législations, le gouvernement du Québec s'efforce dès lors de rendre plus démocratique la vie municipale :

«/.../ en 1968 on accorde le droit de vote à tous les résidents âgés de 18 ans et plus et on étend l'éligibilité, en maintenant toutefois les droits acquis des personnes morales (compagnies, etc.) qui ne perdent leur droit de vote qu'en 1978. La loi de 1968 fixe également les critères de rémunération des élus en fonction de la taille des municipalités, puis celle de 1978 reconnaît les partis politiques municipaux et pourvoit au remboursement d'une partie des dépenses électorales. » (Linteau et al., $1986: 501)$ 
C'est donc dans ce contexte qu'en 1980 la Loi 105 sur la démocratie municipale introduit des mécanismes de participation des citoyens. Cette réforme visait même à juxtaposer à la démocratie classique de représentation une forme plus moderne impliquant davantage les citoyens : la démocratie de participation. Le gouvernement du Québec entendait ainsi favoriser au maximum l'accès à l'information municipale afin qu'un dialogue accru se développe entre les élus locaux et les citoyens. «Ces réformes visent essentiellement à rendre davantage responsables les élus municipaux et à favoriser la participation la plus large possible des citoyens à l'administration des affaires qui les touchent de près.» (Québec, 1978 :3)

\section{Le projet de loi sur la démocratie municipale}

La réforme sur la démocratie municipale se voulait ainsi très ambitieuse en matière de communication avec les citoyens. Dès 1978, le projet du ministre Guy Tardif identifiait d'ailleurs deux grands problèmes en matière d'information aux citoyens :

«/.../ malgré des efforts louables de la part de certaines municipalités, l'information aux citoyens pose encore, règle générale, deux ordres de problèmes : le premier ayant trait à l'accessibilité à l'information et le second concernant la vulgarisation nécessaire des documents municipaux.»

Le gouvernement, soucieux de favoriser la participation la plus large possible des citoyens aux affaires de leur municipalité, estime impérieux de s'attaquer le plus rapidement possible à ces deux ordres de problèmes.» (cité par Baccigalupo, $1990: 213)$

21 Pour ce faire, le projet de réforme sur la démocratie municipale propose, entre autres, la mise sur pied de conseils de quartier, l'obligation de tenir une période de questions à chaque séance du conseil municipal, la diffusion obligatoire en matière budgétaire et la modification des matières pouvant faire l'objet d'un référendum municipal.

La proposition visant la création de conseils de quartier était identifiée comme la " clé de voute » de cette réforme. Dans l'esprit de la proposition, le conseil de quartier devenait :

«... un instrument de formation des habitants d'un quartier, une structure permettant une meilleure circulation de l'information et une façon d'encourager les citoyens à se prendre eux-mêmes en charge. » (Union des municipalités du Québec, 1986 :77)

Malheureusement, la Loi sur la démocratie municipale, adoptée en 1980, ne retient pas la proposition des conseils de quartier en raison de la résistance des élus locaux peu enclins à « partager » leurs ressources de pouvoir. Baccigalupo (1990) relate :

«Ce projet souleva de très vives oppositions chez les élus qui le rejetèrent massivement au nom de la suprématie du conseil municipal et de l'efficacité du processus décisionnel, au nom de la tutelle jugée déjà trop lourde, ainsi qu'au nom de la tradition nord-américaine en matière d'administration des villes. En vérité, les élus locaux repoussèrent ce projet tout simplement parce que les associations de citoyens sont très souvent perçues par les pouvoirs nota biliaires comme de véritables 'irritants', sinon comme la source d'un pouvoir concurrentiel, voire même d'un contre-pouvoir. » (1990:225)

D'autre part, un des points forts de la réforme sur la démocratie municipale a été l'introduction obligatoire de la période de questions lors des assemblées du conseil municipal. Cependant, les modalités d'application de la loi étant laissées à la discrétion des élus municipaux, ces derniers ont eu tendance à interpréter la législation dans un sens restrictif. À titre d'exemple, à Montréal, la plus importante ville du Québec, le 
règlement d'application de la loi fait en sorte que les Montréalais peuvent dorénavant poser des questions à l'administration municipale, celle-ci se réserve toutefois le droit de répondre quand elle le veut et même de ne pas répondre du tout, sans en donner les raisons (Baccigalupo, 1990 :218). En 1991, autre exemple, la ville de Sillery, en banlieue de Québec, adopte un nouveau règlement sur le déroulement des séances du conseil municipal qui limite à une heure la période des questions et à deux minutes le temps alloué à chaque intervenant pour sa première question. Toute infraction peut entraîner l'expulsion de la salle du conseil et une amende de 100 \$ (Le Soleil, 9-4-1991).

Quant à la disposition touchant la diffusion obligatoire de l'information en matière budgétaire, la Loi 105 impose les mesures suivantes :

$\mathrm{Au}$ moins quatre semaines avant que le budget ne soit déposé devant le conseil pour adoption, le maire fait rapport de la situation financière de la municipalité au cours d'une séance du conseil.

Le maire traite des derniers états financiers, du dernier rapport du vérificateur et du programme triennal d'immobilisations, des indications préliminaires quant aux états financiers de l'exercice précédent, celui pour lequel le prochain budget sera fait, et des orientations générales du prochain budget et dans le cas des cités et villes du prochain programme triennal d'immobilisations.

28 Le texte du rapport du maire est distribué gratuitement à chaque adresse civique dans la municipalité. En plus ou au lieu de cette distribution, le conseil peut décréter que le texte soit publié dans un journal diffusé dans la municipalité.

29 Si ces nouvelles mesures sont en elles-mêmes très positives, elles souffrent néanmoins de quelques déficiences. En effet, l'information pèche souvent par un manque de vulgarisation ou par une mise en valeur excessive des gouvernements municipaux (Lavigne, 1993).

30 Enfin, pour ce qui est de la modification des thèmes pouvant faire l'objet d'un référendum, il faudra attendre au 1er janvier 1988 pour voir adopter la Loi sur les élections et les référendums dans les municipalités. Le système municipal prévoit alors la possibilité, et quelquefois même l'obligation, de recourir au mécanisme référendaire afin de faire adopter officiellement un règlement, une résolution ou une ordonnance municipale, notamment en matière d'annexion, de regroupement, de zonage et d'emprunt (Baccigalupo, 1990 :228).

31 Cependant, les scrutins référendaires font encore figure d'exception au Québec. Entre autres, ils ne s'appliquent pas dans les cas des villes de Montréal et de Québec, en vertu de leurs chartes spéciales. En plus, même lorsqu'ils sont décisionnels, comme dans le cas des emprunts municipaux, la fréquence de leur utilisation est rarissime.

"Ces consultations référendaires ramènent aussi sur le tapis la question déjà bien connue des coûts qui y sont rattachés pour la municipalité à laquelle il faut ajouter l'absence des dispositions législatives sur le financement des organisations mises en place pour défendre ou contrer le projet. » (Quesnel, $1992: 7$ )

De fait, l'observation des pratiques de communication gouvernementale entourant certains référendums municipaux tend à démontrer un déséquilibre des forces en matière d'information et de publicité, à la faveur des exécutifs gouvernementaux (Lavigne, 1993).

34 Malgré cela, certains maires de municipalités plus populeuses suggèrent d'amender la loi actuelle afin de limiter le recours au référendum. On souhaite qu'un registre comporte la 
signature de $10 \%$ des gens habiles à voter pour la tenue d'un référendum, au lieu de la règle actuelle de $5 \%$ (Le Soleil, 7-5-1994).

\section{La Loi d'accès et le palier municipal}

En 1982, par ailleurs, le Gouvernement du Québec adopte sa Loi sur l'accès aux documents des organismes publics et sur la protection des renseignements personnels (Loi 65). La Commission d'étude qui a proposé la loi y voit un complément nécessaire aux libertés fondamentales et un apport capable de contrer toute tendance d'un gouvernement de quelque niveau à la dissimulation et à la propagande.

«Sans accès aux faits, sans information, la liberté d'opinion est vidée de sa substance. /.../ La qualité de la participation à la vie politique dépend de la qualité de l'information. Cette information ne peut être triée ou sélectionnée par des hommes qui sont appelés à être jugés. » (Québec, 1981 :5)

Pour les municipalités, la principale conséquence de la Loi d'accès a été d'élargir l'accessibilité du public aux documents détenus par ces institutions. Toutefois, étant donné qu'une législation municipale antérieure avait rendu disponible à chaque citoyen la plupart des documents relatifs à la vie corporative des municipalités par un plus grand accès aux archives (Loi 39), la Loi 65 n'a pas entrainé de "révolution» dans le monde municipal (Baccigalupo, 1990 :216). Elle n'a finalement fait que préciser l'accessibilité d'un plus grand nombre de documents et introduit la notion de protection des renseignements personnels.

En revanche, elle a aussi identifié de nombreuses restrictions au droit d'accès en matière de:

- relations intergouvernementales ;

- négociations entre organismes publics ;

- renseignements économiques ;

- relations de travail et négociations de contrats ;

- sécurité publique et administration de la justice ;

- opinions juridiques et textes réglementaires ;

- décisions administratives ou politiques ;

- renseignements sur les processus de vérification.

Selon Emard (1986:47), malgré toutes les nouvelles dispositions légales, il ressort que le droit à l'information municipale demeure fort incomplet et qu'il incite très peu les citoyens à s'informer. En faisant exception de la Loi 65, l'analyste identifie une série de lacunes en la matière :

- l'absence d'un répertoire qui pourrait codifier toutes les lois et règlements municipaux afin de les rendre plus accessibles pour consultation;

40 - l'absence d'articles spécifiant les délais requis pour l'obtention d'informations;

41 - l'absence d'un mécanismes de recours ou d'appel dans le cas de refus de pourvoir ;

42 - l'absence d'articles quant à l'accessibilité aux documents visuels et sonores par et pour la municipalité ; etc.(1986:48)

Emard (1986:48) dénonce aussi l'absence d'articles concernant la disponibilité ou la diffusion des ordres du jour, comptes rendus et procès-verbaux des réunions du comité exécutif et des comités pléniers lorsque ceux-ci existent. 


\section{Conséquences sur le système municipal actuel}

Les mesures en matière d'accès à l'information et de consultation découlant de la réforme sur la démocratie municipale et de la Loi d'accès, comme nous venons de les exposer, n'ont pas toujours rencontré leurs objectifs initiaux. En effet, à la suite des débats qui suivirent le dépôt des projets, il s'est produit plusieurs glissements entre le projet initial et la réforme réalisée.

Dans ce contexte, comme nous l'avons postulé au début de cet article, l'expérience québécoise n'a pas changé de façon substantielle les ressources de pouvoir des principaux acteurs du système municipal.

Pour les élus, l'information municipale demeure toujours une ressource légitime de l'exercice du pouvoir. À ce titre, les élus de l'équipe au pouvoir prétendent qu'elle est une ressource gouvernementale qui doit servir d'accompagnement à leurs actions et réalisations. Dans la pratique, elle justifie bien souvent la marginalisation des élus qui ne font pas partie de l'équipe politique majoritaire. Ainsi, en dépit de l'avènement des partis politiques locaux dans les municipalités de 20000 habitants et plus, le silence entoure toujours la question de l'opposition au sein des conseils municipaux.

«Il ne suffit pas en effet, à la loi, de permettre l'accès d'une mince opposition au conseil municipal pour que, du même coup, la démocratie fleurisse de façon resplendissante. Encore faut-il que cette opposition soit en mesure d'effectuer honnêtement son travail. Ce qui suppose l'octroi obligatoire et officiel de moyens : libre accès aux bureaux, à l'information interne, droit de disposer d'un personnel de recherche et de secrétariat, etc. » (Baccigalupo, 1990 :235)

Tout au plus, les trois plus grandes villes du Québec, Montréal, Laval et Québec ont obtenu de l'Assemblée nationale que soit votée en 1984 une loi qui accorde aux partis d'opposition des services de secrétariat et un budget de recherche.

De leur côté, les fonctionnaires municipaux ne sont pas devenus plus « ouverts » envers les intermédiaires (journalistes et groupes d'intérêt) et les citoyens, même avec l'implantation de structures de communication dans plusieurs villes.

Le sondage mené en 1988 par l'Union des municipalités du Québec (UMQ) et la Fédération professionnelle des journalistes du Québec (FPJQ) tend à démontrer qu'il existe toujours beaucoup d'incompréhension vis-à-vis du travail des journalistes. De fait, ce sondage avance que plus de la moitié des journalistes estiment éprouver de la difficulté à recueillir de l'information auprès des élus et des fonctionnaires municipaux. Le rapport conjoint $\mathrm{UMQ} / \mathrm{FPJQ}$ traduit en ces termes la rivalité entre le pouvoir politico-administratif et celui de la presse :

«Il y a une incompréhension profonde et parfois une véritable rivalité entre le pouvoir politique et le pouvoir parallèle qu'est la presse. Entre les deux survivent les fonctionnaires municipaux et en particulier les communicateurs. » (UMQ/FPJQ, $1988: 10)$

50 A propos de la perception du rôle des communicateurs par les journalistes, le sondage révèle que même s'ils les aident habituellement à rejoindre le meilleur interlocuteur, les communicateurs municipaux « jouent souvent ou très souvent le rôle d'écran protecteur pour l'administration » (UMQ/FPJQ, $1988: 8)$.

1 En ce qui concerne l'usage de la Loi d'accès, les journalistes y recourent peu en raison, notamment, de la lenteur du processus. 
De leur côté, les groupes d'intérêt constituent des intermédiaires très actifs au palier municipal. Au Québec, pour la plupart des gouvernements municipaux, les groupes sont perçus comme des pourvoyeurs de services socioculturels ou économiques. Les échanges sont davantage favorisés avec les groupes à caractère social et économique à titre de « relais» entre le gouvernement et les citoyens. En ce sens, les gouvernements municipaux entretiennent des discours pompeux sur leur reconnaissance et leur appui technique à certains groupes communautaires. Avec les groupes plus idéologiques, par contre, les relations demeurent plus souvent qu'autrement conflictuelles, bien que ces groupes ne constituent que très rarement une menace réelle pour le pouvoir local.

D'autre part, les modalités d'accès à l'information et de consultation ont modifié les ressources de pouvoir des citoyens de façon plutôt théorique. Dans la pratique, les « simples » citoyens recourent peu à la Loi d'accès (Stanton, 1991) et participent peu aux audiences publiques. Selon Quesnel (1986:88), les mécanismes institutionnels de consultation et de participation sont demeurés peu nombreux et n'ont pas satisfaits les citoyens.

"Associés à quelques comités où ils sont minoritaires, aux côtés des représentants d'autres milieux tels les milieux des affaires et les milieux des spécialistes, les citoyens constatent que leurs intérêts n'y sont pas pris en compte et que ces instances ne sont que consultatives. » $(1986: 87)$

54 En fait, avance Des gagné (1986:6), l’objectif de démocratisation du système municipal par la participation n'a pas permis de donner aux citoyens le contrôle (ou un certain contrôle) de leur devenir collectif en leur permettant de prendre une part effective à l'ensemble (ou à un ensemble) des décisions d'ordre public. En revanche, les élus en ont tiré du capital politique en offrant la participation aux usagers et aux citoyens tout en misant d'avance sur les multiples avantages qu'ils en retirent: image démocratique améliorée, cautionnement de leurs actions, excuses justifiées pour retarder des prises de position délicates, récupération d'éléments plus menaçants en dehors du système qu'à l'intérieur, etc. (Baccigalupo, 1990 :207).

Au Québec, la Loi sur la démocratie municipale de 1980 et la Loi d'accès de 1982 constituent de bons témoins d'un discours sur l'idéal démocratique.

Malheureusement, force nous est de constater que plusieurs mesures en matière d'accès et de consultation des citoyens mis à l'ordre du jour lors de la présentation de ces lois ont été retirées ou ont subi des glissements substantiels sans que l'on ait rajusté le discours et les objectifs poursuivis. De telle sorte que les mesures adoptées n'ont pas réussi à changer de façon substantielle les ressources de pouvoir des principaux acteurs du système municipal. Tout au plus, les nouvelles mesures en matière d'accès et de consultation des citoyens ont favorisé l'institutionnalisation de l'information municipale non pas au profit des citoyens, mais au profit du pouvoir politico-administratif.

En premier lieu, parce que les citoyens ont l'impression d'être protégés par ces mesures, même s'ils s'en prévalent que très peu.

Ensuite, parce que les élus locaux en ont retiré une image démocratique améliorée, sans pour autant changer véritablement leurs pratiques de gestion.

Enfin, parce qu'à l'image du modèle développé dans les organisations commerciales, les structures de communication des municipalités ont rapidement opté pour un modèle basé sur le contrôle de l'information plutôt qu'un modèle de transparence, fondé sur l'accès à l'information et la consultation des citoyens. Dans une telle perspective, il n'est 
pas étonnant que, depuis la fin des années 80 , le discours sur l'information municipale québécoise porte désormais beaucoup plus sur ses vertus stratégiques que sur ses vertus démocratiques.

\section{BIBLIOGRAPHIE}

ANDREW, Caroline et al., L'information sur le logement à Hull, Recherches sociographiques, XVI, 3, 1975, pp. 375-383.

BACCIGALUPO, Alain,, Système politique et administratif des municipalités québécoises, Montréal, Les Editions Agence d'Arc, 1990.

BEAUNEZ, Roger et KOHN, Francis, La démocratie locale, un préalable : l'information dans la cité, Paris, Les Editions ouvrières, 1975, 239 p.

BEAUNEZ, Roger et Max DEJOUR, La commune, le conseil municipal et les citoyens. Pouvoir local et démocratie, Paris, Tema/Adels, 1976,160 p.

BECQUART-LECLERCQ, « Culture organisationnelle et gestion locale », Politiques et management public, vol. 6, nº 1, mars, 1988, pp. 21-60.

BLACK, E. R., P. SNOW, « The Political Agendas of three Newspapers and City Governments ", Canadian Journal of Communication, vol. 8, $\mathrm{n}^{\circ}$ 2, 1982, pp. 11-25.

BLAIS, André, Jean CRETE, «La presse et la politique municipale », Politique, no 2, automne, 1982, pp. 41-67.

CASTAGNET, Michel, « Les règles du jeu d'une communication locale », Territoires-Correspondance municipale, mai, 1991, pp. 6-9.

CHANEL, Alain, « L'élaboration du plan d'occupation des sols : un moment privilégié de l'action municipale? », Albert MABILEAU et André-Jean TUDESQ, L'information locale, Paris, Pedone, 1980, pp. 54-61.

COLLARD, André, Odette LUPIEN et Françoise PENVEN Le guide de la communication municipale, Ottawa, Association des officiers municipaux en communication, 1982, 74 p.

COMET, Henri-Michel, « La communication territoriale : nouvelle ressource ou alibi pour la démocratie ", Revue française d'administration publique, n 58, avril-juin, 1991, pp. 249-252.

DEMERS, François, « L'accès aux médias pour les causes impopulaires. Le cas du Rassemblement populaire de Québec, de 1977 à 1981 », Communication Information, vol. 9, n² 2,1988, pp. 85-108.

DEMERS, François, « L'acquisition d'information par le moyen des médias lors d'une campagne électorale, le cas des élections municipales de Québec en novembre 1989 », Inédit, 1990.

DESGAGNE, Pierre, La participation des citoyens et la réforme de la démocratie locale: une analyse critique de la réforme, Québec, Ministère des Affaires municipales, 1986.

DION, Stéphane, «Politique et gestion municipale », Politique et management public, mars, 1984, pp. 3-24.

DION, Stéphane, La politisation des mairies, Paris, Economica, 1986, 217 p. 
EMARD, Claude, Les politiques d'information municipale dans les grandes villes : une illustration de Montréal 1960-1985, Université du Québec à Montréal, mémoire de maîtrise en science politique, $1986,154 \mathrm{p}$

GAGNON HOSSON, Lise, Les règles de base de la communication municipale, Québec, Association des communica-teurs municipaux du Québec, 1987, 34 p.

GARNIER, J-P. et D. GOLDSCHMIDT, La comédie urbaine ou la société sans classe, Paris, Maspéro, 1978.

GAUTHIER, Gilles, « L'argumentation stratégique dans la communication politique : le débat télévisé L'Allier-Bertrand », Politique, n 17,1990, pp. 114-141.

GERSTLE, Jacques, La communication politique, Paris, PUF, 1992,127 p.

GONTCHAROFF, Georges, L'information municipale, pédagogie de la participation, Paris, Centre national d'art et de la culture Georges Pompidou, 1980, 80 p.

GONTCHAROFF, Georges, « La démocratie locale en question », Revue internationale d'action communautaire, 13/53, printemps 1985, pp. 119-124.

GONTCHAROFF, Georges, «Propagande ou expression des citoyens. Quelle politique de communication, avec quels moyens », Territoires-Correspondance municipale, janvier-février, 1989, pp. 29-31.

GONTCHAROFF, Georges, «Au delà de la gestion. La démocratie de participation », TerritoiresCorrespondance municipale, janvier-février, 1990a, pp. 42-50.

GONTCHAROFF, Georges, « Moderniser et démocratiser la vie locale », Territoires-Correspondance municipale, mai, 1990b, pp. 53-60.

GONTCHAROFF, Georges (1991), « Les départements communiquent », Territoires-Correspondance municipale, janvier, pp. 40-46.

GRAVEL, Robert J. et Robert PETRELLI, « Directeur général : une fonction complexe », Municipalité, mai, 1989a, pp. 2-4.

GRAVEL, Robert J. et Robert PETRELLI « Directeur général : une position clé », Municipalité, juin, 1989b, pp. 20-22.

GREMION, Catherine De la participation à la communication, Revue française d'administration publique, n 58, avril-juin, 1991, pp. 271-274.

JOSEPHE Pascal, Jean-Pierre MURET L'information, communiquer avec le citoyens dans la commune, Paris, Syros, 1983, 242 p.

LAGROYE, Jacques, «L'information et le pouvoir local », Albert MABILEAU et André-Jean TUDESQ, L'information locale, Paris, Pedone, 1980, pp. 71-86.

LAVIGNE, Alain, L'information des gouvernements municipaux en contexte électoral : système d'information et bulletin municipal de trois villes de la Communauté urbaine de Québec, Québec, Université Laval, thèse de doctorat en science politique, 1993, 441 p.

LINTEAU, Paul-André et al., Histoire du Québec contemporain. Le Québec depuis 1930. Montréal, Boréal, 1986, 737 p.

MALLET, Etienne, Information-participation dans votre commune, Editions du Moniteur, Paris, $1978,155 \mathrm{p}$.

MIEGE, Bernard, La société conquise par la communication, Grenoble, Presses universitaires de Grenoble, 1989, 226 p. 
PADIOLEAU, Jean-G., « Les villes-acteurs », Territoires-Correspondance municipale, décembre, 1990, pp. 55-60.

PAILLIART, Isabelle, «L'information municipale : fondement d'une nouvelle démocratie politique? », Raison Présente, no 61, 1982, pp. 69-79.

PAILLIART, Isabelle, « Municipalités : de l'information à la communication », Le bulletin de L'IDATE, n 14, avril, 1984,pp. 167-175.

PAILLIART, Isabelle, « Les villes communiquent », Médiaspouvoirs, no 15, juillet-août, 1989, pp. 135-145.

PAILLIART, Isabelle, «Quand le local s'épuise dans le municipal. La politique dans les télévisions locale par câble », Mots, n 25, décembre, 1990, pp. 81-84.

PAILLIART, Isabelle, Les territoires de la communication, Grenoble, Presses universitaires de Grenoble, 1993, 279 p.

PLOURDE, Marc « Information ou complaisance », La Revue Municipale, août-septembre, 1988a, p. 4.

PLOURDE, Marc, «Le maire au centre de la communication municipale », La Revue Municipale, août-septembre, 1988b, pp. 9-10.

PLOURDE, MARC, « Les élus comprennent mal notre travail », La Revue Municipale, aoûtseptembre, 1988c, pp. 11-12.

PLOURDE, Marc, « Presse et élus devront apprendre à respecter leur rôle », La Revue Municipale, août-septembre, 1988 d, pp. 14 et 21.

QUEBEC, La revalorisation du pouvoir municipal, Québec, Ministère des Affaires municipales, 1981

QUEBEC, Information et liberté. Rapport de la Commission d'étude sur l'accès du citoyen à l'information gouvernementale et sur la protection des renseignements personnels, Québec, Ministère des Communications, 1981.

QUENNEVILLE, Benoît, La presse écrite locale et l'ordre du jour des affaires publiques municipales en Mauricie, Université Laval, mémoire de maîtrise en sciences sociales, 1984, 167 p.

QUESNEL, Louise, « La démocratie municipale au Québec », Politique, n 9,1986, pp. 61-97.

QUESNEL, Louise, Le gouvernement local : plus qu'une administration, Texte ronéotypé, Université Laval, 1988, $31 \mathrm{p}$.

QUESNEL, Louise, Serge BELLEY Partis politiques municipaux. Une étude de sociologie électorale, Montréal, Editions Agence d'Arc, 1991,285 p.

QUESNEL, Louise, « La santé de la démocratie locale », Municipalité, novembre, 1992, pp. 4-7.

REVELIN, Jacques, « Pour tourner le regard des citoyens vers leur municipalité », Municipalité, février, 1983, pp. 22-23.

SIMON, Jean-Paul, « Communication municipale : la double contrainte », Médias pouvoirs, $\mathrm{n}^{\circ} 22$ avril-mai-juin, 1991, pp. 37-51

STANTON, Julie, « Malgré une augmentation de $52 \%$ des demandes, la Loi sur l'accès reste peu connue », Le bulletin des communications, janvier-février, 1991, p. 33.

UNION DES MUNICIPALITES DU QUEBEC, Rapport de la commission d'étude sur les municipalités, Montréal, UMQ, 1986. 
UNION DES MUNICIPALITES DU QUEBEC/ FEDERATION PROFESSIONNELLE DES JOURNALISTES DU QUEBEC, Sondage auprès des dirigeants municipaux et des journalistes sur les relations entre les municipalités et la presse, (rapport conjoint), mai, 1988,.

\section{RÉSUMÉS}

L'enjeu démocratique a toujours constitué une valeur phare au palier municipal. Au Québec, cet idéal a été renforcé par la Loi sur la démocratie municipale de 1980 et la Loi sur l'accès aux documents des organismes publics et sur la protection des renseignements personnels de 1982. Dans son article, l'auteur présente certains glissements de l'expérience québécoise, plus précisément en matière d'accès à l'information et de consultation des citoyens.

Democratic issue has al-ways been an important ideal at the municipal level. In Québec, this value was reinforced by the Law on Municipal Democracy in 1980 and the Law on Access to Official Documents in 1982. In this article, the author examines some faults in Québec experience, more specificly regarding information access and citizens consultation.

\section{AUTEUR}

\section{ALAIN LAVIGNE}

Alain Lavigne est chargé de cours en communication et en journalisme à l'Université Laval, à Québec et à l'Université du Québec, à Rimouski. Sa thèse de doctorat en science politique (1993) porte sur l'information des gouvernements municipaux québécois en contexte électoral. Ses principaux champs de recherche sont la communication publique des municipalités, la communication politique médiatisée et la presse régionale. 